ISSN 2447-9071

doi https://doi.org/10.36414/rbmc.v6i15.49

Contato para correspondência:

Frank Sousa Castro

E-mail:

knarfcastro@gmail.com

Conflito de interesse: Não

Financiamento: Recursos próprios

Recebido: 30/06/2020

Aprovado: 10/08/2020

\section{Doenças de notificação compulsória no laboratório escola da pontifícia universidade católica de goiás no ano de 2018}

\section{Compulsory notification diseases in the laboratory school of pontifical catholic university of goiás in 2018}

\author{
Aline Freitas de Siqueira', Frank Sousa Castro ${ }^{1,2}$ \\ ${ }^{1}$ Pontifícia Universidade Católica de Goiás - PUC Goiás \\ ${ }^{2}$ Faculdade da Polícia Militar - FPM
}

\section{Resumo}

A importância da notificação de doenças garante que medidas de prevenção e controle sejam desencadeadas no caso de doenças graves ou com potencial de disseminação de surtos. Toda e qualquer ocorrência de surto, mesmo que não esteja na lista de notificação compulsória, deve ser obrigatoriamente solicitado ao Ministério da Saúde. Além da lista de doenças de notificação compulsória outros meios de informação podem e devem ser adquiridos e trabalhados para a melhoria da qualidade das atividades de vigilância epidemiológica no nosso país. Oestudo é de caráter observacional retrospectivo de cunho descritivo epidemiológico. Foi realizado no laboratório escola de análises clínicas da Pontifícia Universidade Católica de Goiás, Goiânia-GO, coletando dados a respeito das doenças de notificações compulsórias mais prevalentes no ano de 2018. Foram identificados os grupos de doenças mais prevalentes no laboratório PUC Goiás, como a Sífilis, que compôs mais da metade dos casos, com 64,3\% dos achados e chagas com 12,8\% dos casos. Todas as doenças do presente estudo estão situadas na lista de doenças de notificação compulsória nacional.

Palavras-Chave: Epidemiologia, Notificação de Doenças, Síflis.

\begin{abstract}
The importance of disease is to ensure that prevention and controlmeasures are triggered in the case of serious diseases or those with the potential to spread outbreaks. Any and all outbreaks, even if they are noton the mandatorynotification list, must be referred to the Ministry of Health. In addition to the list of compulsory notification diseases, other means of information can and must be acquired and worked on to enhance and give quality to epidemiological surveillance activities in our country. The study is of a retrospective Observational character of an epidemiological descriptive nature. It was carried out at the clinical analysis laboratory school of the Pontifical Catholic University of Goiás, Goiânia-GO, collecting data on the most prevalent compulsory notification diseases in 2018. The most prevalent disease groups in the PUC Goiás laboratory was identified, such as syphilis, which made up more than half of the cases with $64,3 \%$ of the cases and Chagas disease with 12,8\% of the cases. All the diseases in the present study are on the national list of compulsory reporting diseases.
\end{abstract}

Keywords: Epidemiology, Disease Notification, Syphilis. 


\section{Introdução}

É datado do ano de 1377 o primeiro rol de enfermidades de notificação compulsória. Esse trabalho foi obtido por meio de legislação fundamentada na quarentena, em Veneza' '. Desta forma, a notificação compulsória consiste na ocorrência de determinada doença ou agravo, que deve ser designada às autoridades sanitárias por profissionais da saúde ou qualquer cidadão, para que medidas de intervenção pertinentes sejam tomadas.

As doenças de notificação compulsória (DNC's) constam na lista de agravos de notificação em âmbito mundial, nacional, estadual e municipal. São doenças cujo os agentes causadores são capazes de causar surtos e epidemias, o que exige medidas eficientes de prevenção e controle ${ }^{2}$.

De acordo com a lei $n^{\circ} 6.259 / 75$ a obrigatoriedade da notificação de doenças tornou-se um mecanismo essencial no controle epidemiológico. No Brasil as "Normas Gerais Sobre Defesa e Proteção da Saúde" foram criadas em 1961 pelo decreto $\mathrm{N}^{\circ}$ 49.974-A, onde associavam 45 doenças, viroses humanas e empecilhos do trabalho, de notificação compulsória. As informações eram recebidas por meio das Secretarias Estaduais de Saúde e outros órgãos próprios do Ministério da Saúde e referidas em um Boletim Epidemiológico em um período quinzenal. Contudo só em 1969 se iniciou a notificação sistemática de algumas doenças transmissíveis'.

Devido à proporção continental do Brasil, é insuficiente uma única lista de doenças para todo o território, mesmo com as delimitações de áreas regionais de obrigatoriedade de notificações. Contudo, é necessária uma lista mínima nacional de doenças que apresente informações de relevância para o país. Essas informações têm como objetivo a utilização imediata no local para medidas de controle da população brasileira em situações emergenciais. Estados e municípios devem ser encorajados a elaborar suas listas de notificação compulsória visando uma fortificação dos sistemas locais de Vigilância Epidemiológica (VE), assim podendo ter autonomia para enfrentar os problemas de saúde que possam vir a existir com a realidade de cada área, levando em conta os agravos de relevância regional ou local'.

Ao falar sobre a lista nacional dos agravos de notificação compulsória dever ser claro que a finalidade dessa lista é estabelecer critérios que permitam medir a importância de um agravo de uma perspectiva de vigilância epidemiológica ${ }^{3}$.

Baseado na Lei Orgânica da Saúde, Lei no 8.080, de 1990, a VE é descrita como "um conjunto de ações que proporcionam o conhecimento, a detecção ou prevenção de qualquer mudança nos fatores determinantes e condicionantes de saúde individual ou coletiva, com a finalidade de recomendar e adotar as medidas de prevenção e controle das doenças e agravos"4.
A vigilância era da responsabilidade do Ministério do Trabalho (MT), sendo aplicadas as penalidades previstas na Lei no 6.514, de 1977, da Consolidação das Leis Trabalhistas (CLT), que define a notificação compulsória. Essa prática era realizada através dos profissionais do MT com autoridade de fiscalização do ambiente de trabalho com o objetivo de coagir as empresas a criarem ambientes mais saudáveis e que não cause danos aos seus trabalhadores ${ }^{5}$.

O Sistema de Informação de Agravos de Notificação (SINAN) realiza o registro de doenças de notificação compulsória no país e tem como finalidade transmitir, coletar e disseminar elementos criados rotineiramente pelo sistema de vigilância epidemiológica das três esferas do governo. $\mathrm{O}$ sistema é de rede informatizada e serve de apoio à análise das doenças de notificação compulsória. Atualmente o Sinan está disponível nas versões: Sinan Net, Sinan online e Sinan influenza. O Sinan foi introduzido a partir de 1993, essa introdução foi nas unidades federadas e municípios, não tendo uma coordenação e assistência pelos gestores da saúde. Em 1998 o uso do Sinan foi regimentado. $O$ Sinan vem sendo atualizado regularmente para adaptar-se às necessidades da vigilância em saúde . $^{\text {. }}$

O estudo de prevalência de doenças é designado a deter e prevenir que tanto indivíduos quanto a população em geral sejam acometidos por determinada doença. A importância da notificação de doenças garante que medidas de prevenção e controle sejam desencadeadas no caso de doenças graves ou com potencial de disseminação de surtos ${ }^{4}$.

O Brasil apresenta bastante êxito na vigilância epidemiológica, prevenção e controle de doenças. Em relação ao cenário mundial, o nosso país vem apresentando um avanço nas atividades de saúde pública. Para que esse avanço tenha continuidade deve ser feito uma investigação epidemiológica com o intuito de obter informações necessárias para identificar e saber quais os modos de transmissão e quais grupos estão mais expostos a riscos, com a finalidade de obter medidas de controle para que não ocorra novos casos e surtos ${ }^{8}$.

A lista de DNC's indica quais doenças são de notificação imediata e quais devem ser identificadas a simples desconfiança. O não implemento desta exigência pode comprometer a eficácia das medidas de prevenção e controle disponíveis nos serviços de vigilância epidemiológica. De acordo com a portaria do art. $7^{\circ}$ a DNC deve ser confidencial, só pode ser disseminada fora do âmbito médico sanitário caso traga algum risco a comunidade, mantendo o anonimato dos cidadãos².

A notificação compulsória imediata $(\mathrm{NCl})$ deve ser elaborada em até 24 horas a partir do informe de incidência da doença, agravo ou evento de saúde pública, pelo meio de comunicação mais rápido. A notificação compulsória semanal 
(NCS) deve ser elaborada em até 7 dias a partir do informe de incidência da doença ou agravo ${ }^{9}$.

Recentemente foi publicada uma nova "PORTARIA N ${ }^{\circ} 264$, DE 17 DE FEVEREIRO DE 2020" designa a Lista Nacional de Notificação Compulsória de doenças, agravos e eventualidades de saúde pública em todo o território nacional ${ }^{10}$.

Toda e qualquer ocorrência de surto, mesmo que não esteja na lista de notificação compulsória, deve ser obrigatoriamente solicitado ao ministério da saúde. Ainda que tenhamos elementos importantes na vigilância epidemiológica, não se trata da única e nem a mais importante. Além da lista de DNC outros meios de informação podem e devem ser adquiridos e trabalhados para a melhoria e qualidade das atividades de VE no nosso país. A VE presume a observação de todas as informações sobre o episódio de doenças e de seus fatores com vista a medidas de controle e prevenção'.

Como foi descrito no trabalho, a notificação compulsória é muito importante para a população em geral, pois pode trazer informações essenciais para prevenção de surtos e epidemias de tais doenças. $O$ estudo tem como objetivo de apresentar tanto à população quanto aos acadêmicos interessados uma notificação compulsória de doenças que mais prevaleceram no laboratório escola da PUC Goiás no ano de 2018. Portanto, o objetivo do presente estudo foi identificar quais doenças de notificação compulsória mais prevaleceram no laboratório escola da PUC Goiás no ano de 2018.

\section{Métodos}

O presente estudo foi de caráter observacional retrospectivo de cunho descritivo epidemiológico, foi realizado no Laboratório Escola de Análises Clínicas da Pontifícia Universidade Católica de Goiás (PUC Goiás), Goiânia-GO. O estudo estruturou-se na coleta de dados a respeito das doenças de notificações compulsórias prevalentes no ano de 2018.

A amostra foi composta por 258 prontuários do ano de 2018 que relatavam o diagnóstico de doenças de notificação compulsória. Foram excluídos do grupo amostral quaisquer prontuários que não estivessem corretamente preenchidos. A pesquisa foi aprovada pelo Comitê de Ética e Pesquisa (CEP) sob o número de parecer 608.207.

As informações pertinentes ao presente estudo foram repassadas para uma planilha em uma sala isolada, identificando os prontuários por numerações e coletando as seguintes informações: Idade, sexo, data de atendimento, exame realizado para o diagnóstico e a doença de notificação compulsória.

Os dados foram tabulados em uma planilha no programa EXCEL 2016. Algumas variáveis nominais foram transformadas em categóricas para a continuação das análises estatísticas. Os resultados foram expressos em média, desvio-padrão, frequências, porcentagens, expostos em gráficos e tabelas. Primeiramente, realizou-se uma análise descritiva. Após, utilizou-se o teste ANOVA one way para verificar se houve diferença significativa entre a idade em grupos de diferentes doenças. Para análise dos dados foi utilizado o Software Statistical Package for the Social Sciences (SPSS) versão 25.0. O nível de significância considerado foi de $p \leq 0,05$.

\section{Resultados}

Ao total, 257 indivíduos foram incluídos no presente estudo, no qual $152(59,1 \%)$ eram do sexo masculino e 105 $(40,9 \%)$ do sexo feminino, com média de idade de 41,98 $( \pm 17,82)$ anos. A figura 1 apresenta o gráfico das doenças de notificação compulsória diagnosticadas no Laboratório Escola de Análises Clínicas PUC Goiás, Goiânia-GO. Entre as DNC's, a mais presente nesta população foi a Sífilis, compondo $63,42 \%$ $(\mathrm{n}=163)$ das doenças diagnosticadas, em seguida Chagas $(12,8 \%)$ e Hepatite B $(9,7 \%)$. Os pacientes acometidos por Toxoplasmose, HIV + Hepatite C e Síflis + Hepatite B apresentarem somente um paciente diagnosticado, sendo assim foram excluídos desta análise.

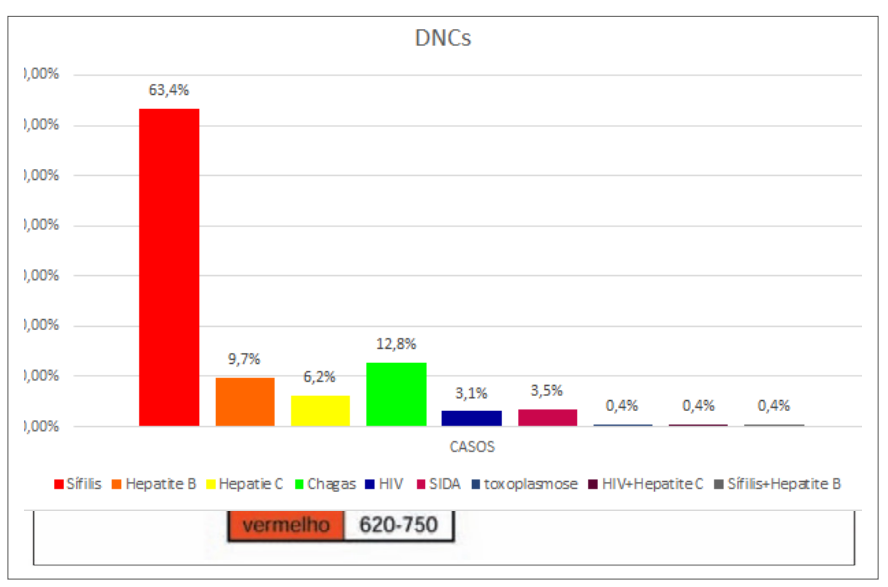

Figura 1. Doenças de Notificação Compulsória diagnosticadas no Laboratório Escola de Análises Clínicas da PUC Goiás, Goiânia-GO $(n=257)$.

Abreviaturas: HIV- vírus da imunodeficiência humana; SIDA- síndrome da imunodeficiência adquirida. Fonte: Elaborado pelo autor (2020).

Em relação a faixa etária mais acometida e quando excluídos os integrantes dos grupos Toxoplasmose, HIV, Hepatite C, Śfilis e Hepatite B observou-se que houve diferença significativa da média de idade entre grupos. Foi identificado que o 
grupo que teve a média de idade mais baixa foi o de pacientes com HIV $(36,00 \pm 10,84)$ e o grupo com a média de idade mais alta foi o de diagnosticados com Chagas $(65,64 \pm 8,67)$, valor de $\mathrm{p}<0,001$ (Tabela-1).

Tabela 1. Comparação da média de idade entre grupos.

\begin{tabular}{ccccc}
\hline Doenças & Média* $^{*}$ & Desvio-padrão & Mínimo & Máximo \\
\hline Sífilis & 36,64 & 15,79 & 0 & 82 \\
Hepatite B & 46,48 & 15,20 & 13 & 71 \\
Hepatite C & 46,44 & 20,16 & 0 & 70 \\
Chagas & 65,64 & 8,67 & 44 & 93 \\
HIV & 36,00 & 10,84 & 19 & 48 \\
SIDA & 38,33 & 10,01 & 23 & 52 \\
\hline
\end{tabular}

Legenda: HIV- vírus da imunodeficiência humana; SIDA- síndrome da imunodeficiência adquirida. Valores relacionados a idade do paciente apresentados em média, desvio-padrão, mínimo e máximo. Realizado o teste ANOVA one way. * ${ }^{*}$ Considerou-se $p$-valor $<0,05$.

Tabela 2. Frequência de indivíduos acometidos por faixa etária.

\begin{tabular}{cccccc}
\hline DNC's & $\begin{array}{c}\mathbf{0 - 1 9} \\
\text { anos }\end{array}$ & $\mathbf{2 0 - 2 9}$ anos & $\begin{array}{c}\mathbf{3 0 - 3 9} \\
\text { anos }\end{array}$ & $\begin{array}{c}\mathbf{4 0 - 4 9} \\
\text { anos }\end{array}$ & $>\mathbf{5 0}$ anos \\
\hline Sífilis & $12(7,4 \%)$ & $57(35,0 \%)$ & $29(17,8 \%)$ & $30(18,4 \%)$ & $35(21,5 \%)$ \\
Hepatite B & $1(4,0 \%)$ & $3(12,0 \%)$ & $4(16,0 \%)$ & $6(24,0 \%)$ & $11(44,0 \%)$ \\
Hepatite C & $2(12,5 \%)$ & $0(0 \%)$ & $1(6,3 \%)$ & $2(12,5 \%)$ & $11(68,8 \%)$ \\
Chagas & $0(0 \%)$ & $0(0 \%)$ & $0(0 \%)$ & $3(6,1 \%)$ & $31(93,9 \%)$ \\
HIV & $1(12,5 \%)$ & $1(12,5 \%)$ & $2(25,0 \%)$ & $4(50,0 \%)$ & $0(0 \%)$ \\
SIDA & $0(0 \%)$ & $2(22,2 \%)$ & $3(33,3 \%)$ & $3(33,3 \%)$ & $1(11,1 \%)$ \\
\hline
\end{tabular}

Legenda: DNC's (doenças de notificação compulsória).

A tabela-3 apresenta análise descritiva dos números de casos de DNC nos meses de 2018. O mês com maior número de casos foi o de novembro, compondo $15,2 \%(n=39)$ das DNC de 2018. Já o com menor números de casos foi o de dezembro, relatando somente duas $(0,8 \%)$ notificações.

Tabela 3. Número de casos de DNC nos meses do ano de 2018 $(n=257)$.

\begin{tabular}{ccc}
\hline Meses & Frequência & Porcentagem \\
\hline Janeiro & 27 & $10,5 \%$ \\
Fevereiro & 13 & $5,1 \%$
\end{tabular}

\begin{tabular}{lll} 
Março & 9 & $3,5 \%$ \\
Abril & 19 & $7,4 \%$ \\
Maio & 22 & $8,6 \%$ \\
Junho & 21 & $8,2 \%$ \\
Julho & 24 & $9,3 \%$ \\
Agosto & 30 & $11,7 \%$ \\
Setembro & 28 & $10,9 \%$ \\
Outubro & 23 & $8,9 \%$ \\
Novembro & 39 & $15,2 \%$ \\
Dezembro & 2 & $0,8 \%$ \\
\hline
\end{tabular}

Os diagnósticos das DNC foram distribuídos pelos meses dos anos, no qual através dos testes Qui-quadrado e razão de verossimilhança não foi apresentado associação entre os meses do ano com o tipo de doença diagnosticada ( $p=0,141 ; p=0,552$ ).

\section{Discussão}

O presente estudo foi baseado na análise das DNC's diagnosticadas pelo laboratório escola da PUC Goiás no ano de 2018. Observou-se que a Sífilis foi a DNC mais presente nessa amostragem, compondo mais da metade dos diagnósticos, perfazendo $63,4 \%$ dos casos. Um fato relevante na pesquisa foi a média de idade $(36,00 \pm 10,84$ anos) dos portadores de HIV, já pacientes portadores com sorologia positiva para chagas foi de $(65,64 \pm 8,67$ anos) prevalecendo na população idosa.

No mês de novembro constatou-se $15,2 \%(n=39)$ de casos totais notificados, sendo responsável pelo maior número de diagnósticos de DNC's. O fechamento dos resultados no final do ano promove medidas de controles de incidência mensal e anual nos laboratórios, de forma que não tenham nenhuma alteração na estrutura epidemiológica. Esses achados contribuem em instrumentos de informações, como a quantidade de pessoas atendidas, ocorrência de surtos no laboratório durante o mês, quantidade de materiais utilizados para exames durantes o mês, busca ativa de casos e dados.

O Brasil vive um período de aumento nos casos de Sífilis nos últimos anos. A notificação compulsória de Sífilis adquirida foi instituída por meio da portaria $n^{\circ} 2.472$, de 31 de agosto de 2010. De acordo com o Ministério da Saúde, os dados do boletim epidemiológico de 2018 apontaram 158.051 casos de Sífilis adquirida (taxa de detecção de 75,8 casos/100.000 habitantes). A população mais afetada no país são mulheres, principalmente as negras e jovens, na faixa etária de 20 a 29 anos $^{11}$. 
Com base nos dados do Laboratório da PUC Goiás, a taxa de incidência de Sífilis foi bastante elevada comparando com a taxa nacional, tendo assim em vista que essa doença tem bastante relevância entre as DNC's. No total de 257 pacientes incluídos no presente estudo, a Síflis foi a doença mais presente nessa amostra, em relação a faixa etária da Sífilis notouse que ocorreu em indivíduos de 20 a 29 anos (35,0\%), tendo assim um resultado semelhante aos casos apresentados no boletim epidemiológico da Sífilis no Brasil, que obteve a faixa etária de indivíduos entre 20 e 29 anos, seguidos por aqueles na faixa entre 30 e 39 anos de idade ${ }^{11}$.

Os casos suspeitos da doença de chagas aguda, devem ser notificados e estabelecido de acordo com a Portaria SVS/MS n ${ }^{\circ}$ 104, de 25 de janeiro de 2011. A região com maior prevalência é região Norte $(82,0 \%)$, e com a menor proporção na região centro-oeste (3,2\%). De acordo com o boletim epidemiológico no território nacional entre os anos de 2012 e 2016 foram registrados no SINAN 19.914 casos suspeitos de doença de chagas aguda e 5,9\% de casos confirmados, já na população estudada observou-se que $12,8 \%(n=31)$ apresentou sorologia positiva para chagas, sendo o segundo agravo mais prevalente dentre as doenças analisadas no presente estudo. Levando em consideração que a região centro-oeste tenha tido a menor proporção de casos, o estudo teve um resultado bastante eminente. Entre os casos do boletim epidemiológico 55,8\% eram homens. A faixa etária mais acometida no boletim epidemiológico de chagas foi de 20 a 49 anos, divergente dos resultados do presente estudo, pois foi o grupo que teve a média de idade mais alta, ocorrendo em indivíduos com mais de 50 anos $(93,9 \%)^{12,13}$.

Outra DNC importante nesse estudo e que foi o terceiro agravo mais prevalente dentre as doenças analisadas foi a hepatite B e em seguida a hepatite $C$. De acordo com as análises da população estudada no presente estudo, $(9,7 \%)$ dos pacientes apresentaram hepatite $B$ e $(6,2 \%)$ hepatite $C$. No Brasil, de acordo com o boletim epidemiológico, no período de 2018 foram notificados 13.922 casos de hepatite B e 26.167 casos de hepatite $C$. Os casos de hepatites virais vêm caindo ao decorrer dos anos de acordo com o ministério da saúde e no presente estudo pode-se perceber que o número de casos é menor em comparação aos outros. De acordo com outros estudos verificou-se que no período de 2008 a 2018 a taxa de detecções das regiões Sul, Norte, sudeste e Centro-Oeste foram superiores à taxa nacional em ambas as hepatites. A prevalência é maior em homens. Em relação à faixa etária de outros estudos, a prevalência de hepatite $B$ em 2018 foi maior entre pessoas de 30 a 49 anos e na hepatite $C$ a faixa etária foi de 55 a 59 anos, nos dez anos de análise houve um aumento na taxa de detecção com destaque em indivíduos de 60 anos ou mais. Já no presente estudo a faixa etária com maior número de casos foi a de pessoas com mais de 50 anos. Como pôde-se observar, os casos de hepatite B e C, tiveram a faixa etária equivalente aos casos apresentados pelo boletim epidemiológico de hepatites virais ${ }^{14}$.

As seguintes DNC analisadas no presente estudo foi HIV e SIDA. De acordo com as análises, (3,5\%) dos pacientes incluídos no presente estudo apresentaram ter SIDA, e $(3,1 \%)$ apresentaram ter HIV. No ano de 2018 foram notificados pelo Sinan, 43.941 casos de infecção pelo HIV no Brasil, já em relação a SIDA, a taxa de detecção vem diminuindo nos últimos anos, no ano de 2018 foram registrados 37.161 casos. Diante desses resultados pode-se constatar que tanto no presente estudo quanto em outros estudos, os casos notificados não são crescidos em comparação de outras doenças, tendo assim pontos favoráveis a população. Foi notificado no Sinan maior casos em indivíduos do sexo masculino tanto em SIDA quanto em HIV. Em relação a faixa etária de outros estudos, observou-se que a maior casos de infecção pelo HIV, encontram-se na faixa de 20 a 34 anos e em SIDA em indivíduos com 25 a 39 anos de idade e que no presente estudo a faixa etária de HIV e SIDA foram entre 30 a 39 anos, tendo assim resultados similares e diante desse desfecho pode-se perceber que a população jovem é a mais acometida ${ }^{15}$.

Os grupos acometidos por Toxoplasmose, HIV, Hepatite C, Síflis e Hepatite B apresentaram somente um paciente diagnosticado em ambos os grupos. Tendo em vista que não teve nenhuma relevância para o presente estudo.

Outro aspecto importante analisado nesse estudo foi que a maior parte dos casos de doenças de notificação compulsória acometeram em indivíduos homens. As diferenças de morbimortalidade entre homens e mulheres são amplamente conhecidas. Os homens são mais acometidos por diversas situações e diferenças nos padrões de comportamento, facilitando a incidência de doenças infectocontagiosas ${ }^{17}$.

\section{Conclusão}

O sistema de notificação compulsória é essencial para o monitoramento da situação de saúde do país, estados e municípios. Com tais informações sobre a situação das DNC's fica mais acessível a elaboração de estratégias que propõem a minimização e controle de tais doenças. Tal fato se faz presente nas doenças notificadas pelo Laboratório Escola de Análises Clínicas PUC Goiás no ano de 2018, podendo assim constatar que essas doenças que estão presente no nosso dia a dia possam ser controladas pelos órgãos reguladores.

Através dos dados coletados foi possível analisar que determinados agravos se tornam necessários a integração 
de práticas e monitorização com finalidade de simplificar a prevenção e controle das doenças notificadas e garantir desenvoltura no fluxo de informações de doenças de interesse a saúde pública.

\section{Referências}

1. Teixeira MG, Penna GO, Risi JB, Penna ML, Alvim MF, Moraes JC, et al. Seleção das doenças de notificação compulsória: critérios e recomendações para as três esferas de governo. Inf. Epidemiol. 1998;7(1):7-28.

2. Silva GA, Oliveira CMG. O registro das doenças de notificação compulsória : a participação dos profissionais da saúde e da comunidade. Rev Epidemiol Control Infect. 2014;4(3):215-20.

3. Hammann EM, Laguardia J. Reflexões sobre a vigilância epidemiológica: mais além da notificação compulsória. Inf Epidemiológico Sus. 2000;9(3):211-9.

4. Sousa SPO, Mascarenhas MDM, Silva MCB, Almeida RAM. Conhecimento sobre doenças e agravos de notificação compulsória entre profissionais da Estratégia Saúde da Família no município de Teresina, estado do Piauí, Brasil - 2010. Epidemiol Serv Saúde. 2012;21(3):465-74.

5. Brasil. Orientações para Ações de Vigilância Epidemiológica. J Chem Inf Model. 2013;53(9):1689-99.

6. Ministério da Saúde. Sistema de Informação de Agravos de Notificação - SINAN: normas e rotinas. Brasília: Editora do Ministério da Saúde, 2006. Disponpivel em: <http://bvsms.saude.gov.br/bvs/publicacoes/ sistema_informacao_agravos_notificacao_sinan.pdf $>$. Acesso em: 20 abr. 2019

7. Hospital Universitário Júlio Muller. Informe epidemiológico., 2005. Disponível em: <http://www2.ebserh. gov.br/documents/17018/2570194/Boletim+Infoma tivo+NVE+HUJM+no.+01_2015.pdf/bb201ed3-820d455b-9adb-2492118536b5>. Acesso em abr. 2019.

8. Ministério da Saúde. Guia de vigilância epidemiológica. 6. ed. Brasília: Editora do Ministrio da Saúde, 2005. Disponível em: <http://portal. anvisa.gov.br/documents/33916/388729/Guia_ Vig_Epid_novo2.pdf/99464018-d6d1-486b-853b9871d6eff16f?version=1.0>. Acesso em 20 abr. 2019.

9. Secretaria de saúde do Ceará. Nota técnica, 2017. Disponível em: <https://www.saude.ce.gov.br/wpcontent/uploads/sites/9/2018/06/nota_tecnica_agravos_notificacao_dez2017_ok.pdf>. Acesso em: 20 abr. 2020.

10. Ministério da Saúde. Portaria n. 204, de 17 de fever- eiro de 2016. Disponível em: <http://portalarquivos2. saude.gov.br/images/pdf/2018/abril/25/Portaria-n--2014-de-17--Fevereiro-2016.pdf >. Acesso em: 20 abr. 2019.

11. Ministério da Saúde. Boletim Epidemiológico Sífilis 2019. Disponível em: <https://portalarquivos2.saude. gov.br/images/pdf/2019/outubro/30/Boletim-S--filis2019-internet.pdf>. Acesso em: 20 abr. 2019.

12. Ministério da Saúde. Doença de Chagas aguda e distribuição espacial dos triatomíneos de importância epidemiológica, Brasil 2012 a 2016. 2019;50:1-10. Disponível em: <http://portalarquivos2.saude.gov.br/ images/pdf/2019/janeiro/23/2018-025.pdf>. Acesso em: 20 abr. 2019.

13. Ministério da Saúde. Vigilância em Saúde no Brasil 2003/2019: da criação da Secretaria de Vigilância em Saúde aos dias atuais. Bol Epidemiológico. 2019;50:1154. Disponível em: <http://www.saude.gov.br/ boletins-epidemiologicos>. Acesso em 15 jul. 2019.

14. Ministério da Saúde. Hepatites Virais 2019. Boletim Epidemiológico. Disponível em: <http://www.aids. gov.br/pt-br/pub/2019/boletim-epidemiologico-dehepatites-virais-2019>. Acesso em: 15 jul. 2019.

15. Ministério da Saúde. Boletim Epidemiológico HIV / Aids | 2019.

16. Ferreira LF, Carneiro MLC, Neiva LGA, Sampaio BM, Gontijo FM, Bueno $\mathrm{H}$, et al. Perfil epidemiológico dos idosos chagásicos da Unidade de Saúde da Família do bairro Nossa Senhora de Fátima, USF - Nossa Senhora de Fátima, da cidade de Paracatu - MG. Rev Med. 2015;94(2):120.

17. Moura E. Perfil da situação de saúde do homem no Brasil. Fiocruz. 2012. 128 p. Disponível em: <https:// portalarquivos2.saude.gov.br/images/pdf/2014/ maio/21/CNSH-DOC-Perfil-da-Situa----o-de-Sa--dedo-Homem-no-Brasil.pdf>. Acesso em 15 jul. 2019. 